\title{
Nonperturbative Approach to Effective Chiral Lagrangians and Meson Interactions
}

\author{
J. A. Oller* and E. Oset ${ }^{\dagger}$ \\ Departamento de Física Teórica and I. F. I. C., Centro Mixto Universidad de Valencia-C. S. I. C., \\ 46100 Burjassot (Valencia), Spain \\ J. R. Peláez \\ Departamento de Física Teórica, Universidad Complutense, 28040 Madrid, Spain \\ (Received 15 September 1997; revised manuscript received 27 February 1998)
}

\begin{abstract}
We develop a coupled channel unitary approach describing the behavior at higher energies of systems whose low-energy dynamics is given by effective $O\left(p^{2}\right)$ and $O\left(p^{4}\right)$ chiral Lagrangians. Our free parameters are those of the $O\left(p^{4}\right)$ Lagrangian. When applied to the meson-meson interaction, it yields a remarkable agreement with data up to $\sqrt{s} \simeq 1.2 \mathrm{GeV}$, dynamically generating the $\sigma, f_{0}, a_{0}, \rho$, and $K^{*}$ resonances. Further applications are also proposed. [S0031-9007(98)05895-5]

PACS numbers: 12.39.Fe, 11.80.Et, 13.75.Lb, 14.40.-n
\end{abstract}

The effective chiral Lagrangian formalism has become a widespread tool to address the problem of the interaction of Goldstone bosons [1,2]. The most significant example is chiral perturbation theory $(\chi \mathrm{PT})[1]$, which successfully describes the low-energy behavior of the meson-meson interaction. To lowest order, $O\left(p^{2}\right)$, the parameters of the chiral Lagrangian are basically masses and decay constants. The $O\left(p^{4}\right)$ contains several free parameters; for instance, in $\chi \mathrm{PT}, 12$ chiral parameters are needed to describe the meson-meson interaction. In the case of the standard model strongly interacting symmetry breaking sector (SISBS), one needs 13 parameters. The limitations of the perturbative chiral approach are obvious, since one cannot attempt to obtain resonances, and it is constrained to low energies. Therefore, nonperturbative schemes become necessary. This is the case when describing the mesonmeson interaction up to about $1.2 \mathrm{GeV}$, where one finds the $\sigma, f_{0}(980), a_{0}(980)$ in the scalar sector and the $\rho(770), K^{*}(892), \phi(1020)$ in the vector channels. The same happens if one tries to study the expected resonance spectrum of the SISBS at the CERN Large Hadron Collider (LHC).

An attempt to extend the ideas of chiral symmetry to the nonperturbative regime, constructing a unitary $t$ matrix, was done in Ref. [3] using the inverse amplitude method (IAM) [4]. This approach proved efficient in reproducing low-energy data and produced poles in the amplitudes associated with the $\rho$ and $K^{*}$ in the vector channel as well as the $\sigma$ in the scalar channel. It has also been applied to study the SISBS resonances that could appear at the LHC [5]. Since only elastic unitarity was imposed in the IAM, multichannel problems could not be addressed. As a consequence, neither the $f_{0}$ and $a_{0}$ resonances nor the inelasticities could be obtained. A similar problem with coupled channels could also appear in the SISBS, if the top quark couples strongly to longitudinal gauge bosons.
The treatment of coupled channels has proved to be crucial in order to reproduce the basic features of the $f_{0}$ and $a_{0}$ resonances [6]. Another nonperturbative method, using coupled channel Lippmann-Schwinger (LS) equations was done in Ref. [7], using the $O\left(p^{2}\right) \chi \mathrm{PT}$ amplitudes, $T_{2}$. A similar work in the $K-N$ system was carried out in Ref. [8].

The LS equations used in [7] read, in matrix form,

$$
T=T_{2}+\overline{T_{2} G T},
$$

where $T_{2}$ and $T$ are $2 \times 2$ matrices. The channels of the mesonic scalar sector in that work are $\pi \pi, K \bar{K}$ for isospin $I=0$ as well as $K \bar{K}, \pi \eta$ for $I=1$. In Eq. (1) $\overline{T_{2} G T}$ is given by

$$
\begin{aligned}
\left(\overline{T_{2} G T}\right)_{i l}= & i \int \frac{d^{4} q}{(2 \pi)^{4}} \frac{T_{2 i j}(k, p ; q)}{q^{2}-m_{1 j}^{2}+i \epsilon} \\
& \times \frac{T_{j l}\left(q ; k^{\prime}, p^{\prime}\right)}{(P-q)^{2}-m_{2 j}^{2}+i \epsilon},
\end{aligned}
$$

with $k, p$ the four momentum of the initial mesons and $P=p+k$. An important point realized in Ref. [7] is that, if $T_{2}(k, p ; q)$ is separated in an on-shell part plus a residual term, then the latter, when used in the loops of Eq. (2), does not have to be calculated, since it leads only to coupling and mass renormalization. This allows the on-shell factorization of $T_{2}$ and $T$ from Eq. (2) reducing the LS equations to pure algebraic relations:

$$
T=T_{2}+T_{2} \cdot G \cdot T .
$$

Thus, we obtain

$$
T=\left[1-T_{2} \cdot G\right]^{-1} \cdot T_{2},
$$

where $G$ is a diagonal matrix given by

$$
\begin{aligned}
G_{i i}= & i \int \frac{d^{4} q}{(2 \pi)^{4}} \frac{1}{q^{2}-m_{1 i}^{2}+i \epsilon} \\
& \times \frac{1}{(P-q)^{2}-m_{2 i}^{2}+i \epsilon} .
\end{aligned}
$$


A cutoff, $q_{\max }$, in the integral over $|\vec{q}|$ in Eq. (5) was adopted as a regularization method, and $q_{\max }$ was adjusted to the data. With a value of $q_{\max }=0.9 \mathrm{GeV}$, a remarkable agreement was obtained for $J=0$, in the $I=0,1$ channels. However, a simple extrapolation of the model to the $J=1$ channel fails to reproduce the $\rho$ and $K^{*}$ resonances, which, however, are nicely reproduced in the scheme of Ref. [3].

It seems clear that there is important dynamics in the $O\left(p^{4}\right)$ chiral Lagrangian, particularly related to vector mesons, which cannot be generated by the LS resummation. In fact, the resonance saturation hypothesis [9] assumes that the $O\left(p^{4}\right)$ terms are generated through the exchange of resonances.

We propose here a simple and rather general unitary scheme in coupled channels which combines elements of the works in Refs. [3] and [7] that can now be obtained as particular cases. When applied to the meson-meson interaction, this method describes simultaneously all of the channels and reproduces the resonances below $1.2 \mathrm{GeV}$.

With $N$ coupled channels and for a given $(I, J)$, unitarity imposes (recall that $T=T^{T}$ )

$$
\operatorname{Im} T=T \sigma T^{*},
$$

where $\sigma$ is an $N$-diagonal matrix accounting for the phase space in each channel. With the normalization of [10] that we adopt in the following: $\sigma=\operatorname{Im} G$ of Eq. (5). Thus, Eq. (6) can be cast as

$$
\operatorname{Im} G=-\operatorname{Im} T^{-1},
$$

which is more conveniently rewritten as

$$
\begin{aligned}
T & =\left[\operatorname{Re} T^{-1}-i \operatorname{Im} G\right]^{-1} \\
& =T_{2} \cdot\left[T_{2} \cdot \operatorname{Re} T^{-1} \cdot T_{2}-i T_{2} \cdot \operatorname{Im} G \cdot T_{2}\right]^{-1} \cdot T_{2} .
\end{aligned}
$$

The next step is to realize that, although $T$ is certainly a poorly convergent function in the chiral expansion (above $500 \mathrm{MeV}$ for mesons), and particularly close to poles, the function $T_{2} \cdot T^{-1} \cdot T_{2}$ may converge much faster. The results we obtain seem to support this conjecture. (Intuitively one can imagine a function such as $T \sim$ $\tan (x), T_{2} \sim x$, and $T_{2} \cdot T^{-1} \cdot T_{2} \sim x^{2} \cot (x)$ expanded around $x=0$. This expansion of $T$ for values of $x$ around $\pi / 2$, where $T$ has a pole, is very poorly convergent, whereas $T_{2} \cdot T^{-1} \cdot T_{2}$ converges very fast.)

Expanding $T$ within the chiral formalism up to $O\left(p^{4}\right)$, we have

$$
\begin{aligned}
T & \simeq T_{2}+T_{4}+\ldots ; \\
T^{-1} & =T_{2}^{-1}\left(1-T_{4} T_{2}^{-1}+\ldots\right),
\end{aligned}
$$

where $T_{4}$ is the pure $O\left(p^{4}\right) \chi \mathrm{PT}$ amplitude. Therefore,

$$
T_{2} \cdot \operatorname{Re} T^{-1} \cdot T_{2} \simeq T_{2}-\operatorname{Re} T_{4}+\ldots
$$

The above derivation is formal, since $T_{2}$ and $T$ may not be invertible. Indeed, that happens for the $(I, J)=(1,1)$ channel. That is the reason why we write $T$ as in Eq. (8).
In that way, we need only the expansion of $T_{2} \cdot \operatorname{Re} T^{-1}$. $T_{2}$, which can be obtained by continuity and, as it can be seen in Eq. (10), is well behaved. Finally, from Eqs. (8) and (10), we arrive at

$$
T=T_{2} \cdot\left[T_{2}-T_{4}\right]^{-1} \cdot T_{2} .
$$

This, by itself, is an interesting result since, at $O\left(p^{4}\right)$, it is the coupled channel generalization of the IAM [3]. Apart from that, this method has other advantages. Indeed, as it is well known [11], the IAM with a single channel has problems around the Adler zeros in the scalar sector. For instance, Eq. (11) with just one channel yields a double zero. The problem is actually more serious since the expansion of $T^{-1}$ in Eqs. (9) and (10) is meaningless, if $T_{2}=0$ or $T_{2}<T_{4}$. However, the expansion of $T^{-1}$ in Eq. (9) holds as a matrix relation, even if one of the matrix elements of $T_{2}$ vanishes or is smaller than the corresponding one of $T_{4}$. Furthermore, the Adler zeros appear as single zeros with the coupled channel method.

Nevertheless, Eq. (11) requires the complete evaluation of the whole $T_{4}$ matrix, including loops, which is rather involved. Indeed, at present, there are only $O\left(p^{4}\right)$ calculations of meson-meson scattering for $\pi \pi$ and $\pi K$ $[1,12]$. However, inspired in the LS equations, we will obtain a good approximation to Eq. (11), without the complete $O\left(p^{4}\right)$ calculation.

Let us then first reinterpret the LS equations discussed above using Eqs. (8) and (10): It is enough to take $G$ as in Eq. (5), with an appropriate cutoff such that $\operatorname{Re} T_{4} \simeq T_{2} \cdot \operatorname{Re} G \cdot T_{2}$, and we recover Eq. (4). Note that, in this way, we are accounting for the $s$-channel one-loop diagram responsible for the unitarity logarithms. In addition, the freedom in the cutoff allows one to reproduce the relevant counterterm contribution in the meson scalar sector, as shown in [7].

However, such approximations cannot hold in all meson-meson channels, since other $O\left(p^{4}\right)$ counterterms become essential. Therefore, in the present paper, we are also considering the polynomial coming from the $O\left(p^{4}\right)$ tree level contributions that we denote by $T_{4}^{P}$, and we write

$$
\operatorname{Re} T_{4} \simeq T_{4}^{P}+T_{2} \cdot \operatorname{Re} G \cdot T_{2} .
$$

The coefficients of $T_{4}^{P}$ are combinations of the $O\left(p^{4}\right)$ chiral parameters (which are usually denoted $L_{i}$ within $\chi \mathrm{PT}$ and $\alpha_{I}$ or $a_{i}$ in the SISBS).

Note that $T_{4}^{P}$ is not just a vehicle for free parameters. It also ensures that we are considering the most general $O\left(p^{4}\right)$ polynomial structure compatible with chiral symmetry and its breaking. Once we have this chiral structure, the cutoff regularization can now be substituted by any other scheme.

In Eq. (12) one is explicitly neglecting the crossedchannel loop contributions. Although these loops do not yield an imaginary part in the $s$ channel, they do contribute to the real part. However, in the $s$ channel 
TABLE I. We list the values of the $\hat{L}_{i}$ parameters (in units of $10^{-3}$ ) obtained from the fit of our method to meson-meson scattering data.

\begin{tabular}{lccccccc}
\hline \hline$q_{\max }=1.0 \mathrm{GeV}$ & $\hat{L}_{1}$ & $\hat{L}_{2}$ & $\hat{L}_{3}$ & $\hat{L}_{4}$ & $\hat{L}_{5}$ & $\hat{L}_{7}$ & $2 \hat{L}_{6}+\hat{L}_{8}$ \\
\hline Our fit & 0.5 & 1.0 & -3.2 & -0.6 & 1.7 & 0.2 & 0.8 \\
\hline \hline
\end{tabular}
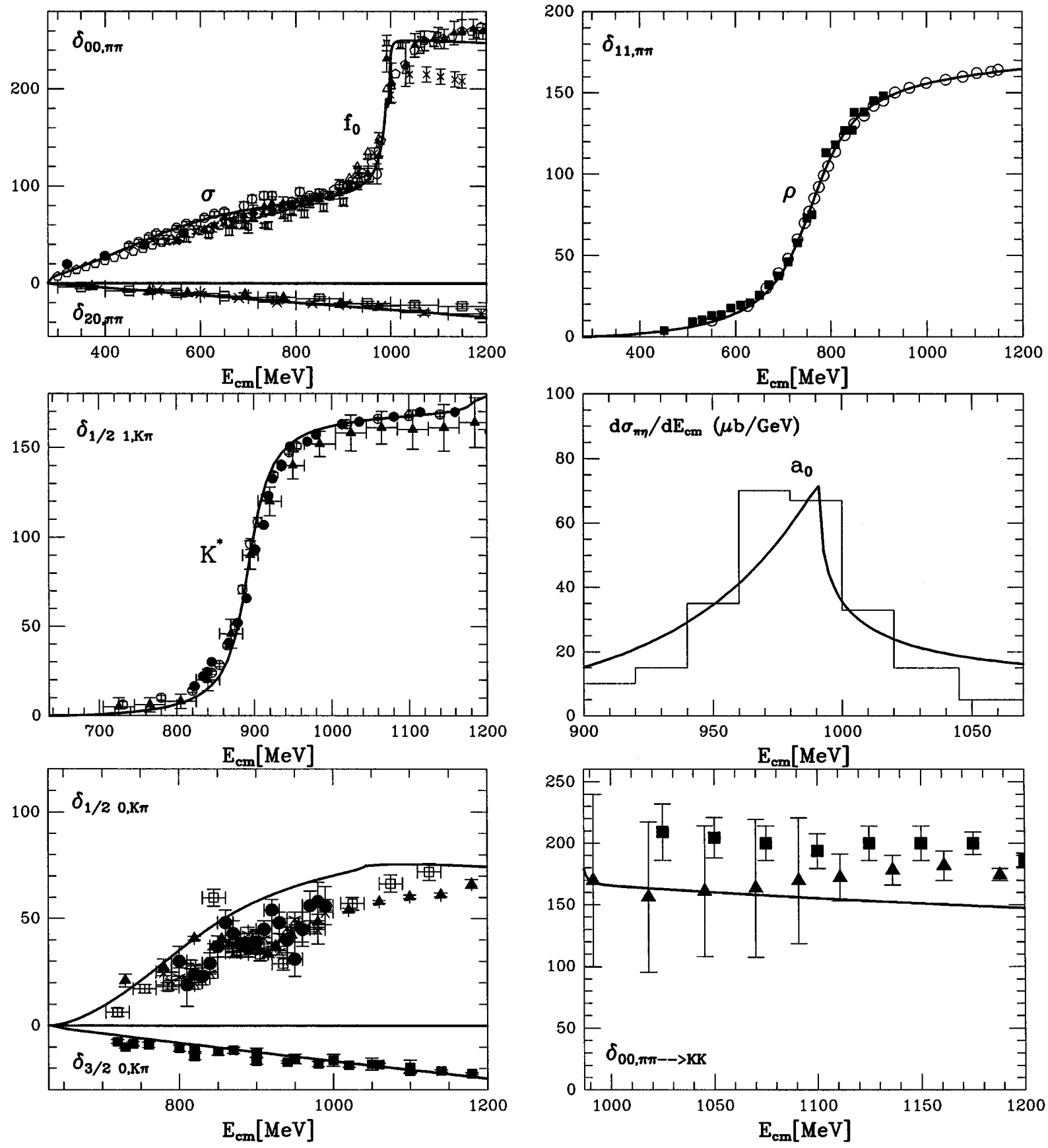

FIG. 1. We display the results of our method for the phase shifts of $\pi \pi$ scattering in the $(I, J)=(0,0),(1,1)$, and $(2,0)$ channels, where the $\sigma, f_{0}$, and $\rho$ resonances appear, together with those of $\pi \pi \rightarrow K \bar{K}$, as well as the phase shifts of $\pi K$ scattering in the $(3 / 2,0),(1 / 2,0)$, and $(1 / 2,1)$ channels, where we can see the appearance of the $K^{*}$ resonance. The results also include the $\pi^{-} \eta$ mass distribution for the $a_{0}$ resonance in the $(I, J)=(1,0)$ channel from $K^{-} p \rightarrow \Sigma(1385) \pi^{-} \eta$. For reference to the data, see [3] and [7], and references therein. 
these contributions have a smooth dependence on the energy and can be effectively reabsorbed in the $L_{i}$ coefficients.

Concerning tadpoles, in the equal mass limit, their finite contributions can be absorbed in the chiral parameters. When considering different masses, it is possible to add and subtract a finite tadpolelike term with an "average" mass. The added part is absorbed in the chiral parameters. The part not absorbed is thus of the order of the difference between physical masses and the average mass, whose value is such that these contributions are minimized.

Then, using Eqs. (11) and (12) we can write our final formula:

$$
T \simeq T_{2} \cdot\left[T_{2}-T_{4}^{P}-T_{2} \cdot G \cdot T_{2}\right]^{-1} \cdot T_{2} .
$$

Our purpose now is to illustrate how well the method that we have developed works in practice. To that aim, we will use it in a fit to meson-meson data, and we will denote the resulting parameters by $\hat{L}_{i}$ (see Table I). The reasons for changing their name are that, first, as we have already commented, part of the effect of crossed-channel terms and tadpoles will be reabsorbed in $\hat{L}_{i}$ and, second, that we have chosen a cutoff regularization.

In Fig. 1 we show the results of our approach, represented by phase shifts of the $\pi \pi \rightarrow \pi \pi, \pi \pi \rightarrow K \bar{K}$, and $K \pi \rightarrow K \pi$ reactions plus a mass distribution for the $a_{0}$ resonance in the $(I, J)=(1,0)$ channel. The curves have been obtained with the fitted parameters given in Table I. The figure shows that the $f_{0}, a_{0}, \rho$, and $K^{*}$ resonances are very well reproduced, and the phase shifts agree remarkably well with data from threshold up to about $\sqrt{s} \simeq 1.2 \mathrm{GeV}$, where the influence of multimeson channels should start to be more relevant. The $\sigma$ meson, around $500 \mathrm{MeV}$ and with a large width, as found in [3] and [7] is also reproduced here.

In summary, we have proposed a unitary scheme in coupled channels that allows us to extend the applicability range of the chiral Lagrangian formalism. We have shown how it can be implemented to $O\left(p^{4}\right)$ using the coefficients of the second order Lagrangian as free parameters. When applied to meson-meson interactions, the scheme is remarkably successful and reproduces the different resonances and data up to about $1.2 \mathrm{GeV}$.

Further applications that we can suggest are $\gamma \gamma \rightarrow$ $M M$ [13], form factors, decay of particles with a pair of mesons in the final state, meson-nucleon interactions, etc. Within the SISBS it can also be very useful to study the coupling of longitudinal gauge bosons to transversal gauge bosons or top quarks. It also seems likely that, with a proper generalization, it could also be applied to nonrelativistic solid-state systems, as high- $T_{c}$ superconductors whose effective Lagrangian formulation is also raising a great interest [14]. The potential of the method to extend the usefulness and advantages of the effective chiral Lagrangian formalism to regions otherwise inaccessible is certainly enormous.

One of us (J. R.P.) thanks the University of Valencia, where this work was carried out, for their kind hospitality. We thank A. Pich and M.R. Pennington for useful comments. J. A. O. acknowledges financial support from the Generalitat Valenciana. This work was partially supported by CICYT under Contracts No. PB96-0753 and No. AEN93-0776.

*Electronic address: oller@condor.ific.uv.es †Electronic address: oset@evalvx.ific.uv.es *Electronic address: pelaez@eucmax.sim.ucm.es

[1] S. Weinberg, Phys. Rev. Lett. 19, 1264 (1967); J. Gasser and H. Leutwyler, Ann. Phys. (N.Y.) 158, 142 (1984).

[2] T. Applequist and C. Bernard, Phys. Rev. D 22, 200 (1980); A. C. Longhitano, Nucl. Phys. B188, 118 (1981).

[3] A. Dobado, M. J. Herrero, and T. N. Truong, Phys. Lett. B 235, 134 (1990); A. Dobado and J. R. Peláez, Phys. Rev. D 47, 4883 (1993); Phys. Rev. D 56, 3057 (1997).

[4] T. N. Truong, Phys. Rev. Lett. 61, 2526 (1988); ibid. 67, 2260 (1991).

[5] A. Dobado, M. J. Herrero, and T. N. Truong, Phys. Lett. B 235, 129 (1990); A. Dobado, M. J. Herrero, and J. Terrón, Z. Phys. C 50, 205 (1991); Z. Phys. C 50, 465 (1991); J. R. Peláez, Phys. Rev. D 55, 4193 (1997).

[6] J. Weinstein and N. Isgur, Phys. Rev. D 41, 2236 (1990); G. Janssen, B. C. Pearce, K. Holinde, and J. Speth, Phys. Rev. D 52, 2690 (1995).

[7] J. A. Oller and E. Oset, Nucl. Phys. A620, 438 (1997).

[8] N. Kaiser, P. B. Siegel, and W. Weise, Nucl. Phys. A594, 325 (1995).

[9] G. Ecker, J. Gasser, A. Pich, and E. de Rafael, Nucl. Phys. B321, 311 (1989); J. F. Donoghue, C. Ramirez, and G. Valencia, Phys. Rev. D 39, 1947 (1989).

[10] F. Mandl and G. Shaw, Quantum Field Theory (Wiley, New York, 1988).

[11] M. Boglione and M. R. Pennington, Z. Phys. C 75, 113 (1997); T. Hannah, hep-ph/9703403.

[12] V. Bernard, N. Kaiser, and U. G. Meißner, Phys. Rev. D 43, 2757 (1991).

[13] J. A. Oller and E. Oset, hep-ph/9706487 [Nucl. Phys. A (to be published)].

[14] H. Leutwyler, Phys. Rev. D 49, 3033 (1994); Helv. Phys. Acta 70, 275 (1997); S.C. Zhang, Science 275, 1089 (1997); C. P. Burgess and A. Lutken, cond-mat/9705216. 